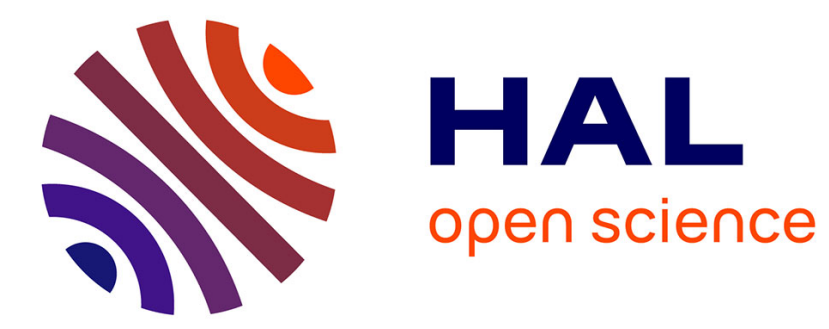

\title{
Model for intensity calculation in electron guns
}

O. Doyen, J.M. de Conto, J.P. Garnier, M. Lefort, N. Richard

\section{- To cite this version:}

O. Doyen, J.M. de Conto, J.P. Garnier, M. Lefort, N. Richard. Model for intensity calculation in electron guns. Journal of Applied Physics, 2007, 101, pp.084914. 10.1063/1.2720091 . in2p3-00144717

\section{HAL Id: in2p3-00144717 https://hal.in2p3.fr/in2p3-00144717}

Submitted on 21 May 2007

HAL is a multi-disciplinary open access archive for the deposit and dissemination of scientific research documents, whether they are published or not. The documents may come from teaching and research institutions in France or abroad, or from public or private research centers.
L'archive ouverte pluridisciplinaire HAL, est destinée au dépôt et à la diffusion de documents scientifiques de niveau recherche, publiés ou non, émanant des établissements d'enseignement et de recherche français ou étrangers, des laboratoires publics ou privés. 


\title{
A new model for intensity calculation in electron guns
}

\author{
O. Doyen, JM. De Conto \\ Fourier ; 53 avenue des Martyrs 38026 GRENOBLE, France. \\ JP. Garnier, M. Lefort, N. Richard \\ Thomson Genlis $S A$; avenue du Général de Gaulle 21110 GENLIS, France.
}

Laboratoire de Physique Subatomique et de Cosmologie - CNRS - IN2P3 - INPG - Université Joseph

\begin{abstract}
The calculation of the current in an electron gun structure is one of the main investigations involved in the electron gun physics understanding. In particular, various simulation codes exist but often present some important divergences with the experiment. Moreover, those differences cannot be reduced because of the lack of physical information in them. We present a simple physical three dimensional model, valid for all kinds of gun geometries. This model presents a better precision than all the other simulation codes and models encountered, and allows the real understanding of the electron gun physics. It is based only on the calculation of the Laplace electric field at the cathode, the use of the classical Child-Langmuir's current density, and a geometrical correction to this law. Finally, the intensity versus voltage characteristic curve can be precisely described with only a few physical parameters. Indeed, we have showed that only the shape of the electric field at the cathode without beam, and a distance of an equivalent infinite planar diode gap, govern mainly the electron gun current generation.
\end{abstract}

\section{INTRODUCTION}

The design of electron guns is nowadays an advanced field for which, more especially, various simulation codes exist. However, progresses are still necessary in order, for example in the CRT domain, to improve the performances of small depth and great angle tubes. The systematic use of codes can then appear to be limited (design time too important, losses in the understanding of the most relevant physical behaviors). We have started (collaboration CNRS-Thomson Genlis SA) a study program that aim at obtaining a precise and simple theoretical model able to make the most relevant parameters appear, contrary to the simulation codes that sometimes seem like "black boxes". More particularly, the knowledge of the current generation is a compulsory step in the whole electron gun physics understanding.

In principle, the calculation of the current requires the solution of Poisson's equations, which are non-linear, and can only be obtained numerically, by creating simulation codes. ${ }^{1-6}$ However, as exposed before, this generally involves complicated procedures. Moreover, the divergences with the experiment are often too important, and cannot be corrected because of the absence of physical models, especially for complex gun geometries. In order to obtain a better understanding of the current characteristics, for instance, its dependence on various factors such as applied voltages and gun geometry, it is preferable to use some simple approximate formulas. Various authors investigated on this kind of models. ${ }^{7-}$ ${ }^{17}$ Nevertheless, most of them are built on empirical statements (for example, Hasker uses a real gun as reference in his calculations and ad hoc formulas). ${ }^{14,15}$ Furthermore, all of these studies are valid only for simple rotationally symmetrical electron guns (succession of two circular hole-drilled electrodes). As a result, guns made of complex holes structures cannot be tackled. And finally, even in those simple cases, the precision obtained is not very satisfying: it can be acceptable on a limited range of currents (10\% of accuracy between 0.5 and $5 \mathrm{~mA}$ for a symmetrical triode), ${ }^{17}$ but it's never satisfying on the whole intensity-voltage characteristic curve. In particular, for high intensities, an important domain to test the life time of a cathode, the results obtained in those studies (and in the simulation codes as well), are far from the experiment.
We present here a new, simple, and accurate physical model of current generation in electron guns, which describes with a very high precision the whole intensity-voltage characteristics (called "drive curve"), including high intensities, for all kind of symmetrical and asymmetrical gun geometries. The accuracy obtained is far better than the one encountered in the other studies and simulation codes. The main important physical parameters at stake are: the electric field on the cathode without beam, therefore without space charge effects, (and more particularly its emissive surface, and electric field value at the cathode centre), the use of the classical one dimension ChildLangmuir's law for calculating the current density, ${ }^{18}$ and a distance $\mathrm{D}$ of an equivalent infinite planar diode gap.

We first present a full analytical model for a simple case, a rotationally symmetrical gun with identical-holes electrodes, in order to understand the main physical tools at stake. The potential in this structure, and then the electric field on the cathode, are calculated analytically thanks to Bessel's functions. The classical Child-Langmuir's law being given for an infinite planar diode, we will show that a correction is required to take into account the finite radius of the beam. We will then give two possibilities for the definition of the equivalent diode distance, the best of them needing an experimental reference. We will finally show that this model presents an accuracy greater than the current simulation codes, by comparing with the experiment and with one simulation program. This code is evoked in the references. ${ }^{3}$

Then, this model being validated in a simple case, we will present its generalization into a numerical model valid for all kinds of gun geometries. In particular, the main differences with the previous model appear in the electric field calculation, and the adaptation of the correction of Child-Langmuir's law.

To sum up, we will show that the beam creation, in terms of current density, is based on very simple laws, and is ruled only by the electric field on the cathode (without beam) and ChildLangmuir's law adapted to a finite beam dimension.

\section{ELECTRON GUN STRUCTURE AND NOTATIONS}

An electron gun is made of a cathode (noted $\mathrm{K}$ ) followed by 
several hole-drilled electrodes (G1, G2 and G3), called grids, into which passes the electron beam. This beam is created by thermoionic emission at the cathode plane $(0, \mathrm{x}, \mathrm{y})$, and is accelerated (and decelerated) through the $\mathrm{z}$ direction of the gun thanks to the Laplace potential (i.e. without beam) formed by the grid voltages. In particular, the resulting electric field at the cathode $\mathrm{E}_{\mathrm{K}}$ that extracts the electrons is influenced only by the first three grids voltages $\left(\mathrm{V}_{\mathrm{G} 1}, \mathrm{~V}_{\mathrm{G} 2}\right.$, and $\left.\mathrm{V}_{\mathrm{G} 3}\right)$, the cathode voltage $\mathrm{V}_{\mathrm{K}}$, and the gun geometry. Its positive part will be considered as the emissive area of the cathode (defined as the zone where the field accelerates the electrons), and, its value at the cathode centre will be noted $\mathrm{E}_{\max }$. The cathode plane is taken as the zero equipotential.

\section{A SIMPLE CASE}

In this part is described the analytical model valid for rotationally symmetric guns with identical-holes electrodes (of radius $\mathrm{R}$ ), as represented in figure 1 ( $\mathrm{r}$ being the radial coordinate).

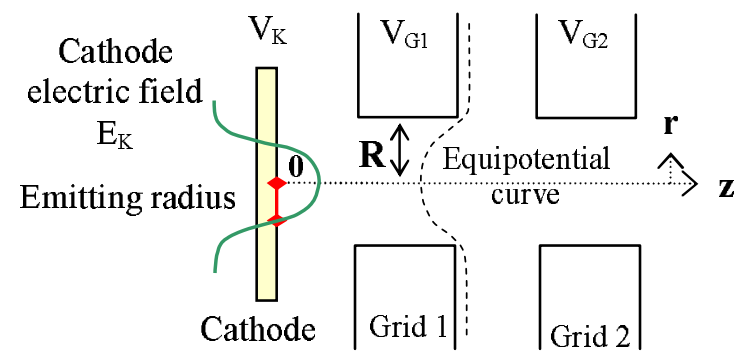

FIG. 1. Description of the structure of the electron gun studied in the following.

This study highlights the main elements involved in the general model (part 2).

\section{A. Analytical potential calculation.}

The electrostatic potential in the beam forming region (without beam) is the first mandatory step for the model. In a general 3D gun, the potential is obtained from numerical calculations like finite elements (see paragraph 2.1). Nevertheless, for cylindrical guns, an analytical tool has been developed and the potential V is written in the following form:

$$
V(r, z)=\int_{-\infty}^{+\infty} K(\omega) I_{0}(\omega r) \exp (j \omega z) d \omega
$$

where $I_{0}$ is the Bessel function of first kind. The detailed calculation of the kernel $\mathrm{K}$ is given in appendix.

The advantage of this development is to get a fast and selfsufficient analytical model using classical tools like Maple or Mathematica, without the need of additional codes. Even limited to cylindrical guns, this permits to get the significant physical parameters of the gun, which could be generalized to any gun shape.

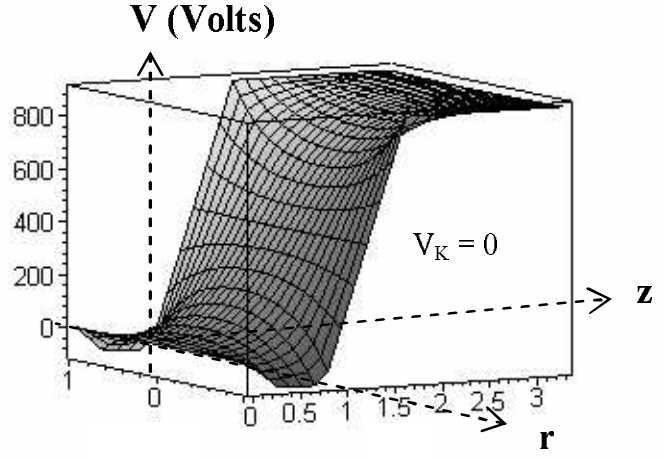

FIG. 2. Variation of the Laplace potential in plane $(r, z)$ (arbitrary units).

We check that the potential calculated (displayed in figure 2) is close to the result provided by a simulation code. This code is evoked in the references. ${ }^{3}$

\section{B. Derivation of the cathode electric field}

By analytical derivation versus $\mathrm{z}$ we obtain the cathode electric field (all the other components are zero):

$$
E_{K z}=E_{z}(r, 0)=\left.\frac{\partial V(r, z)}{\partial z}\right|_{z=0}
$$

Figure 3 illustrates this parameter on the cathode plane for a given gun and voltage set.

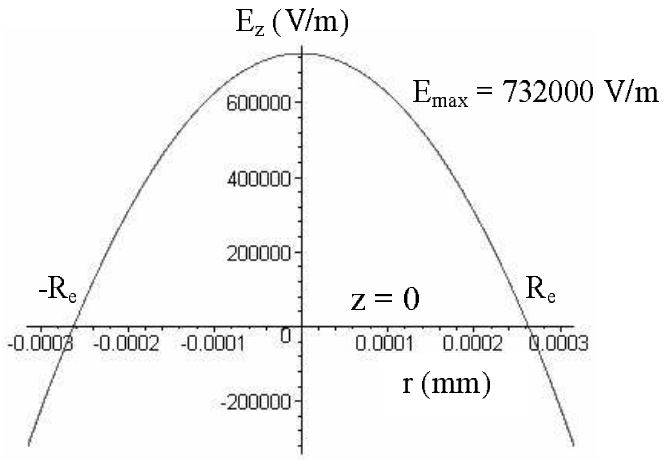

FIG. 3. Cathode electric field for $\mathrm{V}_{\mathrm{K}}-0 \mathrm{~V}, \mathrm{~V}_{\mathrm{G} 1}-0 \mathrm{~V}, \mathrm{~V}_{\mathrm{G} 2}-900 \mathrm{~V}$, and a given gun geometry.

With our notations, the positive part of $\mathrm{E}_{\mathrm{K}}$ defines the emissive zone of the cathode. As a result, for this example, the emitting radius $R_{e}$ is $0.243 \mathrm{~mm}$. Moreover, we verify that $E_{K}$ has a parabolic shape, as used by most of the previous authors. However, we will show in part 2 that for more complicated gun structures, the parabolic approximation cannot be made.

\section{Current density and total current}

To calculate the cathode current density $j_{\mathrm{K}}$ from the cathode electric field $E_{K}$ we propose to use the classical one dimensional Child-Langmuir's law. ${ }^{18}$ We suppose, like many authors, that the system behaves like concentric independent diodes following Child-Langmuir's law. It gives: 


$$
j_{K}(r)=\frac{4 \sqrt{2 e / m} \varepsilon_{0}}{9 \sqrt{D}} E_{K}^{3 / 2}(r)
$$

where $\mathrm{D}$ is the classical diode gap, e the elementary charge, and $\varepsilon_{0}$ the permittivity of vacuum.

By integrating $\mathrm{j}_{\mathrm{K}}$ on the emitting cathode surface (a disc of radius $\mathrm{R}$ ), we obtain the gun intensity:

$$
I=\int_{0}^{R_{e}} 2 \pi r j_{K}(r) d r .
$$

And, by plotting $I=f\left(V_{K}\right)$, we get the current versus voltage characteristic curve called "drive curve".

Obviously, formula (1) is not obvious in our case, because it is valid only for infinite diodes in the radial direction. As a result, some modifications and optimizations are required in order to apply this law to our non-infinite-diode geometry. First, the equivalent diode distance $\mathrm{D}$ has to be defined and work for our geometry. Second, in order to take into account the finite dimension of the beam, Child-Langmuir's law has to be corrected. These two points will be discussed in the next three paragraphs.

\section{Correction of Child-Langmuir's law}

The classical Child-Langmuir's law for the current density is a 1D model and made for two infinite parallel electrodes. When the radius of the beam is finite, this density becomes higher and a small but not negligible correction has to be done. The general case has been studied elsewhere and is given in paragraph 2.3. Nevertheless, a simple correction has been estimated at the beginning of our studies showing that this correction step is mandatory. ${ }^{19}$

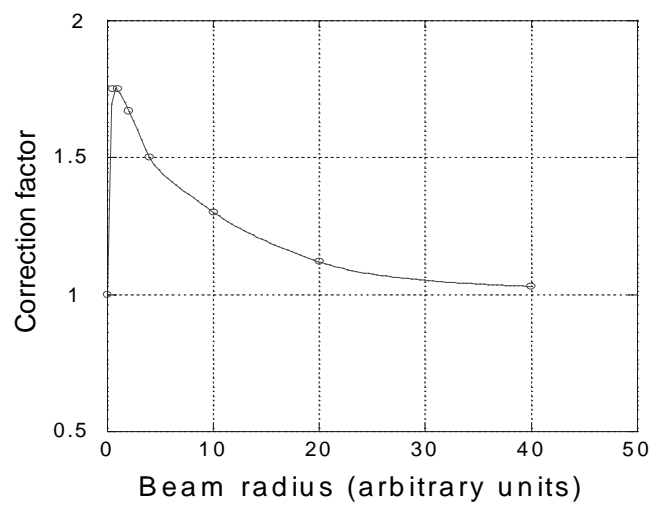

FIG. 4. Correction factor to the Child-Langmuir's law.

The calculation is made for a Thomson gun example. For large radiuses, the Child Langmuir's law remains valid. At low radiuses (small currents), the correction can be up to $70 \%$ (see figure 4).

\section{E. Determination of the equivalent diode gap parameter}

In most previous publications this parameter is chosen constant, what appears to be a good hypothesis for a given geometry, however the assumptions made in these studies are not accurate enough and don't work for complex geometries.
This parameter is not totally understood yet, but we noticed that it depends only on the gun geometry, what is equivalent to say that it depends only on the cathode electric field.

We propose two solutions to tackle this problem: on the one hand, for measured guns, one experimental reference is enough to get the equivalent diode distance. Indeed, in order to get the best precision as possible in comparison with the experiment, for a given gun, we have to chose $\mathrm{D}$ so that :

$$
I\left(V_{K}=0 V\right)=I_{\text {exp }}\left(V_{K}=0 V\right) .
$$

As a result, only the experimental current value at $V_{K}=0 \mathrm{~V}$ is needed to obtain a high precision on the whole characteristic curve. We have noticed that for a given gun, in every grid voltages configurations, this distance remains constant.

On the other hand, for new guns, Ploke's equivalent diode distance concept can be used (cf. paragraph 5). ${ }^{10}$ The precision obtained on the current vs. tension characteristics using this definition is quite satisfying. This is explained in paragraph 4 .

\section{F. Results}

After correcting Child-Langmuir's law and choosing the right parameter $\mathrm{D}$, we compared to the experiment and to a simulation code for two different guns. Gun s1 has small electrode holes in comparison with gun s2, and, the thickness and spacing of the electrodes are significantly different.

For both guns, the coherence with the experiment is very good (precision better than 5\%) for the whole range of intensities, and every type of grid voltage configuration, unlike the simulation code results, as shown in figures 5 and 6 .

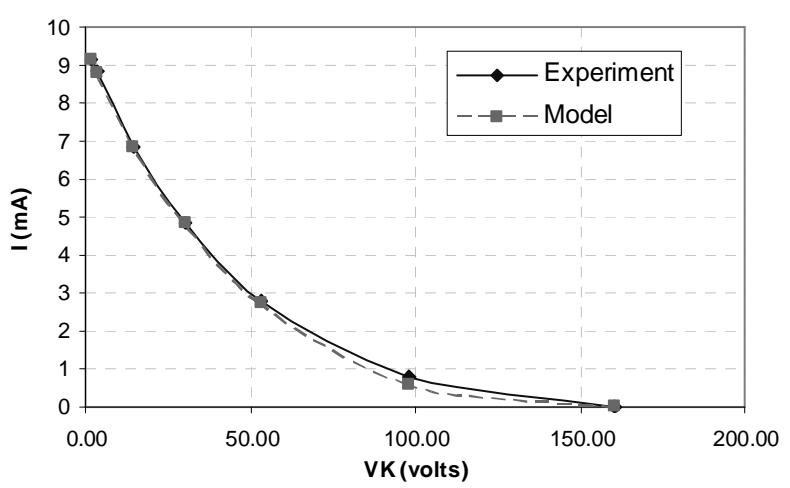

FIG. 5. Model and experiment for current versus voltage characteristic of gun $\mathrm{s} 1, \mathrm{D}-0.283 \mathrm{~mm}$.

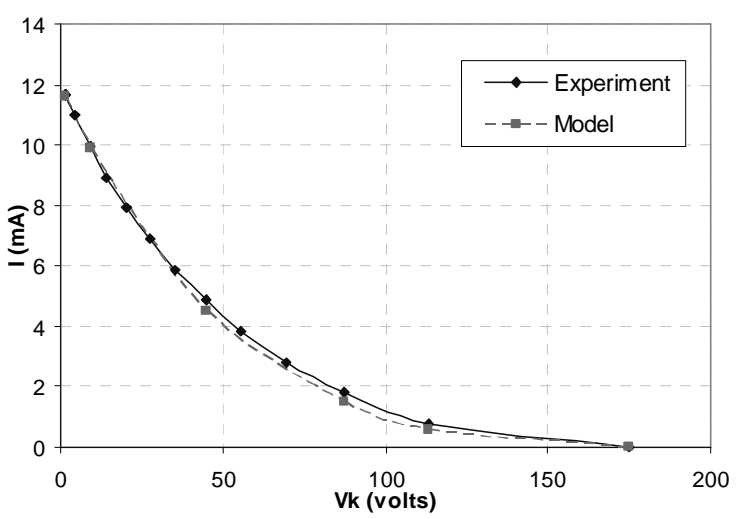


FIG. 6. Model and experiment for current versus voltage characteristic of gun $\mathrm{s} 2, \mathrm{D}-0.392 \mathrm{~mm}$.

This elementary case, solved with simple analytical tools, permits to be aware of the elements at stake for modeling the current generated in an electron gun: the cathode electric field is the key point of the model. Then, a correction applied to the classical one dimensional Child-Langmuir's law, and the choice of an equivalent diode distance lead to a good correlation with the experimental current tension characteristic.

We have set up a simple physical model. Its accuracy is better the simulation code we use. We will now generalize this model to a full three dimension case.

\section{GENERAL CASE}

Although for simple symmetrical guns it is possible to calculate analytically the potential in the structure, and thus the cathode electric field, this parameter cannot be worked out easily for asymmetrical cases, and above all, the main difficulty comes from the fact that it has to be valid for all kinds of geometries. As a result, this calculus will be realized numerically, by simulation.

\section{A. Calculation of the cathode electric field}

Various simulation codes exist which are able to solve Laplace's equation $\Delta \mathrm{V}=0$ in gun structures, and precise in the electrostatics calculations. We will use one of them, ${ }^{3}$ to work out the electric field at the cathode without beam.

In order to know this field for every voltage configuration, the unitary contribution of every single grid is calculated (i.e. the cathode electric field created by 1 Volt on the cathode and OV to the other grids, and so on). We deduce the cathode electric field by superposition, as follows:

$$
\begin{aligned}
& \begin{aligned}
E_{K z}\left(V_{K}, V_{G}, V_{G 2}, V_{G 3}\right) & =V_{K} \cdot E_{K}\left(V_{K}=1, V_{G}=V_{G 2}=V_{G 3}=0\right) \\
& +V_{G 1} \cdot E_{K}\left(V_{G 1}=1, V_{K}=V_{G 2}=V_{G 3}=0\right) \\
& +V_{G 2} \cdot E_{K}\left(V_{G 2}=1, V_{K}=V_{G 1}=V_{G 3}=0\right) \\
& +V_{G 3} \cdot E_{K}\left(V_{G 3}=1, V_{K}=V_{G 1}=V_{G 2}=0\right)
\end{aligned} \\
& \begin{aligned}
(1) \quad \begin{array}{l}
E_{K}\left(V_{K}=50 V, V_{G 1}=\right. \\
\text { represented in figure } 7 \text { for an asymmetrical gun. }
\end{array}
\end{aligned}
\end{aligned}
$$

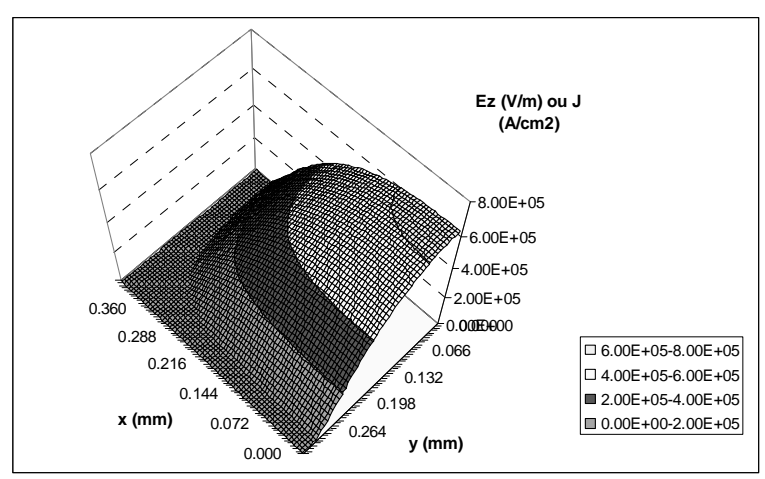

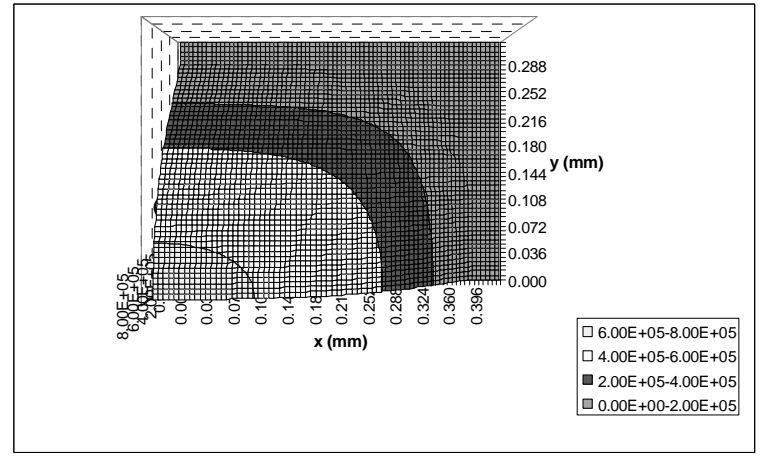

FIG. 7. Representations of one fourth of the electric field $E_{K}\left(V_{K}=50 \mathrm{~V}, V_{G 1}=-190 \mathrm{~V}, V_{G 2}=1220, V_{G 3}=8000 \mathrm{~V}\right)$ on the cathode for an asymmetrical gun.

It has been noticed, by verifying on six different guns, that for every gun, the cathode electric field can be, in first approximation, represented as elliptical iso-field curves with a parabolic profile. However, for a good precision, the parabolic assumption on the cathode electric field profile, often encountered in the literature, cannot be made (see figure 8).

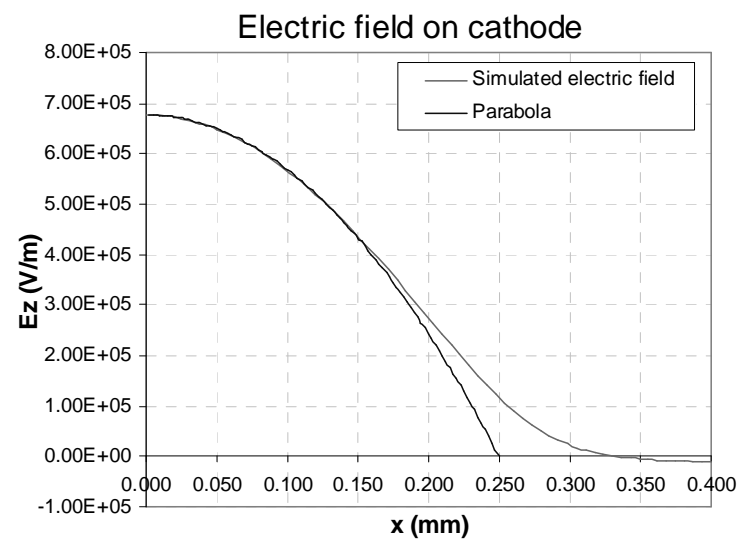

FIG. 8. Comparison between the cathode electric field at full intensity and a parabola, for a given gun

As represented in the previous picture, we noticed that for every gun, the electric field is not parabolic at full intensity (i.e. when $V_{K}=0 \mathrm{~V}$ ), but for small intensities, the parabolic assumption is relevant. Indeed, we show that changing $V_{K}$ corresponds to a simple translation of the electric field with respect to the cathode: if the cathode voltage is decreased by $\Delta V_{K}$, then the cathode electric field (cf. formula (1)) decreases by:

$$
\begin{aligned}
\Delta E_{K}\left(V_{K}, V_{G 1}, V_{G 2}, V_{G 3}\right) & =\Delta V_{K} \cdot E_{K}\left(V_{K}=1 V, V_{G 1}=V_{G 2}=V_{G 3}=0\right) \\
& =\text { constant }
\end{aligned}
$$

Since we have a numerical model, we will not use any approximations, and work with the "real" shape of the electric field.

\section{B. Current density and current}

Like in part 1 , to calculate the cathode current density $j_{K}$ from the cathode electric field $\mathrm{E}_{\mathrm{K}}$ we use the classical one dimensional Child-Langmuir's law: 


$$
j_{K}(x, y)=\frac{4 \sqrt{2 e / m} \varepsilon_{0}}{9 \sqrt{D}} E_{K}^{3 / 2}(x, y) .
$$

The intensity is obtained by summing the current densities multiplied by the elementary surfaces (defined by the numerical mesh) all over the cathode emissive surface:

$$
I=\sum_{i, j} j_{K}\left(x_{i}, y_{j}\right) \Delta x \Delta y
$$

where $\Delta x$ and $\Delta y$ are the steps of our mesh, and $(i, j)$ the indexes of the calculated point.

Like before, first, this law has to be corrected, and then, the equivalent diode distance has to be defined. These two points will be discussed in the next two paragraphs.

\section{New correction of Child-Langmuir's law}

The correction used in part 1 can't be applied here, because the assumptions were carried out for a symmetrical case. The new correction, valid for all cases, comes from a publication of W.S. Koh, L.K. Ang and T.J.T. Kwan. ${ }^{20}$

In this document, it is shown that the link between the classical one dimensional Child-Langmuir's law j(1D) and the corrected 3D law $\mathrm{j}(3 \mathrm{D})$ for a planar diode can be written :

$$
\frac{j(3 D)}{j(1 D)}=1+F^{*} G
$$

where $\mathrm{F}$ and $\mathrm{G}$ are factors depending respectively on the average position of the electrons, and the emissive surface geometry. We then have to multiply the intensity previously calculated by $1+F^{*} G$, where $\mathrm{F}=0.25$, in our case, and $\mathrm{G}$ is known depending on whether we consider an ellipse, a rectangle, and so on, as the emitting surface (for a uniform current density). We can consider that for all the guns, the emissive surface is elliptical, therefore the expression of $\mathrm{G}$ is given by: $:^{20}$

$$
G=\frac{2}{\pi} \frac{E\left(\sqrt{1-(b / a)^{2}}\right)}{b / D},
$$

where $\mathrm{E}(\mathrm{)})$ is the complete elliptic integral of the second kind, a and $b$ are respectively the long and short radius of the ellipse, and $\mathrm{D}$ is the equivalent diode distance.

For a circular emissive surface, this coefficient becomes:

$$
G=\frac{D}{r} \text {, where } \mathrm{r} \text { is the circle radius. }
$$

The current density being uniform in this reference, unlike in our case, we have to calculate the right parameters $a$ and $b$. To do so, we integrate the cathode electric field in the plane $y=0$ for $\mathrm{a}$, and $\mathrm{x}=0$ for $\mathrm{b}$, in order to build equivalent uniform distributions while keeping the maximum field $\mathrm{E}_{\max }$. In plane $\mathrm{y}=0$ we find parameter $\mathrm{a}$ with:

$$
\int_{0}^{\infty} E(x) d x=E_{\max } \cdot a,
$$

and in plane $\mathrm{x}=0$ we find $\mathrm{b}$ with:

$$
\int_{0}^{\infty} E(y) d y=E_{\max } \cdot b .
$$

Choosing $\mathrm{E}_{\max }$ as the value of the uniform distribution appeared to be the best and simplest solution among others.

Figure 9 presents an example of variation of this correction for a given asymmetrical gun: at low currents the correction can be up to $90 \%$.

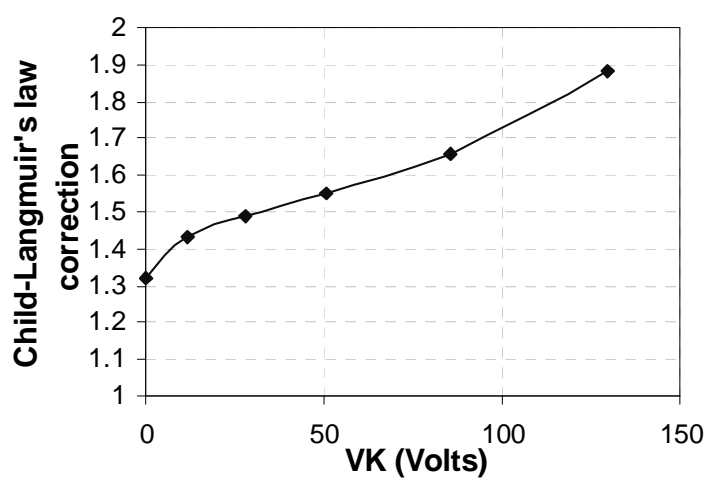

FIG. 9. Variation of the correction factor versus the cathode voltage for a given asymmetrical gun.

We notice that the correction increases with the emitting surface. Moreover, we verify that for every gun, the correction around $0 \mathrm{~V}$ almost doesn't change, and remains close to 1.3 .

\section{Determination of the equivalent diode distance}

In comparison with part III, no modification is made for the determination of this parameter. If we have one experimental reference at $V_{K}=0 V$, then we choose $\mathrm{D}$ so that $I\left(V_{K}=0 V\right)=I_{\exp }\left(V_{K}=0 V\right)$. Like in the simple case of part 2, we have noticed that for a given gun, in every grid voltages configurations, this distance remains constant.

If no experimental value is available, Ploke's equivalent diode distance concept can be used for new guns, ${ }^{19}$ as explained later in part VI.

\section{RESULTS FOR THE GENERAL CASE}

In this part are presented several comparisons with the experiment or/and with a simulation code (same code as before), in order to validate the electric field and the intensity calculations (more particularly, the current vs. voltage characteristics). Moreover, by analyzing those results, many interesting observations were made.

To do so, we have six different guns (gun s1 and gun s2 are symmetrical, whereas the guns a1 to a4 are asymmetrical with complex geometries) at our disposal, that have been tested experimentally and simulated as well.

\section{A. Results related to the cathode electric field calculations}

Let's verify that, for calculating the electric field, the superposition method is accurate, and that the simulation code previously used is coherent with the experiment.

\section{Definition of the "cut-off" notion}

The "cut-off" is the electric state of a gun at the emission limit, that is to say for zero intensity. The cut-off voltage $\mathrm{V}_{\mathrm{Kco}}$ is defined as follows: first, the cathode is set at $+\mathrm{V}_{\mathrm{Koo}}$. Then, the first grid is set to zero. And finally, second grid voltage is adjusted to $\mathrm{V}_{\mathrm{G} 2 \mathrm{co}}$ such as $\mathrm{I}\left(\mathrm{V}_{\mathrm{G} 2 \mathrm{co}}\right)=0$. The drive curve is then obtained by changing $\mathrm{V}_{\mathrm{K}}$ within the domain $\left[0,+\mathrm{V}_{\mathrm{Kco}}\right]$.

For example, in the case of gun a1, a classical cut-off 
configuration is: $\mathrm{V}_{\mathrm{Kco}}=150 \mathrm{~V}, \mathrm{~V}_{\mathrm{Gl}}=0 \mathrm{~V}, \mathrm{~V}_{\mathrm{G} 2 \mathrm{co}}=520 \mathrm{~V}$ and $\mathrm{V}_{\mathrm{G} 3}$ $=3000 \mathrm{~V}$. In the industry, electron guns are not only used in classical cut-off configurations, but also in extreme voltages cases, such as the so called "6MIK" configuration: first we have $\mathrm{V}_{\mathrm{K}}=0 \mathrm{~V}, \mathrm{~V}_{\mathrm{G} 1}=-50 \mathrm{~V}, \mathrm{~V}_{\mathrm{G} 2}=\mathrm{V}_{\mathrm{G} 2 \mathrm{co}}$ and $\mathrm{V}_{\mathrm{G} 3}=3000 \mathrm{~V}$; and then, $\mathrm{V}_{\mathrm{G} 1}$ is set to $0 \mathrm{~V}$ whereas the other voltages remain constant. "7MIK" is also an other configuration similar to $6 \mathrm{MIK}$ except that $\mathrm{V}_{\mathrm{G} 1}=-150 \mathrm{~V}$.

\section{Comparison of the cut-off voltages}

Let us compare in table I the cut-off voltages with the experiment in order to validate the electric field calculated by superposition from simulation codes results.

TABLE I. Modeled and measured cut-off voltages for several guns, and for $\mathrm{V}_{\mathrm{Kco}}-190 \mathrm{~V}$

\begin{tabular}{ccccc}
\hline Voltage & Gun s1 & Gun al & Gun a2 & Gun s2 \\
\hline $\begin{array}{c}\mathrm{V}_{\mathrm{G} 2 \mathrm{co}} \\
\text { (experiment) }\end{array}$ & $1082 \mathrm{~V}$ & $616 \mathrm{~V}$ & $1250 \mathrm{~V}$ & $510 \mathrm{~V}$ \\
$\mathrm{~V}_{\mathrm{G} 2 \mathrm{co}}$ (model) & $1001 \mathrm{~V}$ & $668 \mathrm{~V}$ & $1223 \mathrm{~V}$ & $507 \mathrm{~V}$ \\
\hline
\end{tabular}

Therefore, the superposition method using simulation codes gives cut-off voltages quite close to the experimental values. We verify as well that we have the same accuracy in the 6MIK and $7 \mathrm{MIK}$ cut-off configurations.

\section{Remarks on the emissive surface at full intensity}

A complementary checking of the cathode emissive surfaces permits to notice that, for a given gun, the emissive surface at full intensity is constant for all cut-off configurations $\left(\mathrm{V}_{\mathrm{K}}=150 \mathrm{~V}\right.$ or $\mathrm{V}_{\mathrm{K}}=200 \mathrm{~V}$ or $6 \mathrm{MIK}, 7 \mathrm{MIK}$, etc $\left.\ldots\right)$. This can be easily explained because at full intensity, $\mathrm{V}_{\mathrm{Gl}}=\mathrm{V}_{\mathrm{K}}=0$, and given that G3 has almost no influence on the cathode field (cf. observations at the end of this paragraph), $\mathrm{V}_{\mathrm{G} 2}$ is linearly linked to the electric field (cf. formula (1)):

$$
E_{K}\left(V_{K}, V_{G 1}, V_{G 2}, V_{G 3}\right) \approx V_{G 2} \cdot E_{K}\left(V_{G 2}=1 V, V_{K}=V_{G 1}=V_{G 3}=0\right)
$$

The cathode electric field amplitude is proportional to $\mathrm{V}_{\mathrm{G} 2}$, but the emitting surface remains constant.

Furthermore, this area is higher than the surface of the first grid hole (factor 2.2 for gun s2 and 1.5 for gun a1). But the contribution of the cathode area greater than G1 hole surface is relatively small ( $2 \%$ of the current for gun s2 in $7 \mathrm{MIK}$ conditions).

\section{B. Results related to intensity calculations}

We will first show that the intensity calculated is close to the experiment, for any kind of cut-off configuration, and then show that the precision on the whole of the current vs. voltage characteristics is very good. Moreover, we will also make a comparison with the previous simulation code, and show that this tool his highly inaccurate regarding the drive curves.

For a given gun and equivalent diode distance (defined in classical cut-off conditions), we show that we have a good model for calculating intensities even in extreme cut-off conditions as $6 \mathrm{MIK}$ and $7 \mathrm{MIK}$ (cf. table II).
TABLE II. Modeled and measured intensities at $\mathrm{V}_{\mathrm{K}}-0 \mathrm{~V}$, in $6 \mathrm{MIK}$ and $7 \mathrm{MIK}$ conditions, and for two different guns.

\begin{tabular}{|c|c|c|c|c|}
\hline \multirow{3}{*}{ Condition } & \multicolumn{2}{|c|}{ Gun al } & \multicolumn{2}{|c|}{ Gun s2 } \\
\hline & $\mathrm{I}(0 \mathrm{~V})$ & $\mathrm{I}(0 \mathrm{~V})$ & $\mathrm{I}(0 \mathrm{~V})$ & $\mathrm{I}(0 \mathrm{~V})$ \\
\hline & experiment & model & experiment & model \\
\hline $6 \mathrm{MIK}$ & $1.34 \mathrm{~mA}$ & $1.19 \mathrm{~mA}$ & $1.14 \mathrm{~mA}$ & $0.99 \mathrm{~mA}$ \\
\hline $7 \mathrm{MIK}$ & $6.41 \mathrm{~mA}$ & $6.38 \mathrm{~mA}$ & $5.45 \mathrm{~mA}$ & $5.08 \mathrm{~mA}$ \\
\hline
\end{tabular}

Finally, for every gun and every cut-off configuration, we compare the current versus voltage characteristics to the experiment, and realize that the coherence with the measured curves is extremely satisfying.

Figures 10 and 11 present some examples of comparisons of drive curves on two different guns.

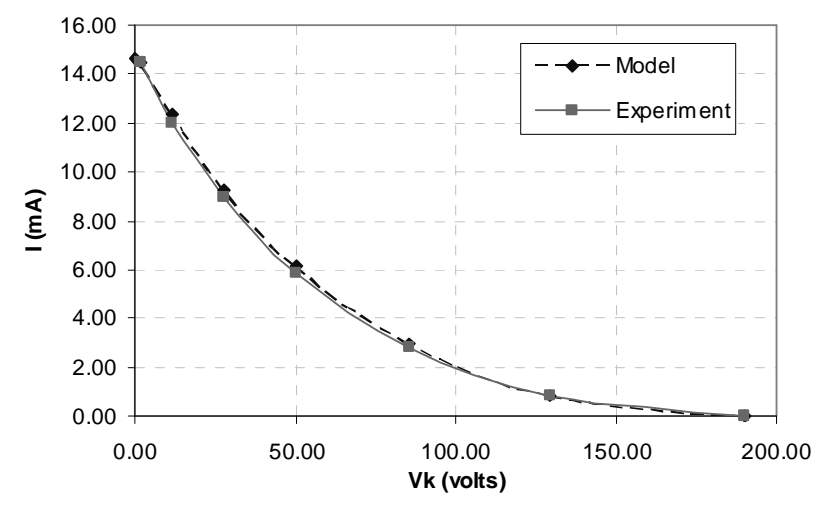

FIG. 10. Drive curve for gun $\mathrm{a} 1, \mathrm{~V}_{\mathrm{K} \text { co }}-190 \mathrm{~V}$, and $\mathrm{D}-0.72 \mathrm{~mm}$.

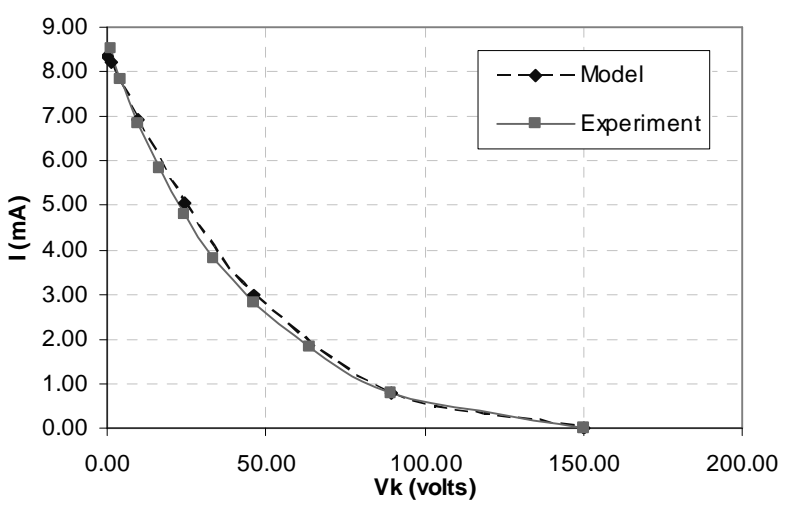

FIG. 11. Drive curve for gun $\mathrm{s} 1, \mathrm{~V}_{\mathrm{Kco}}-150 \mathrm{~V}$, and $\mathrm{D}-0.55 \mathrm{~mm}$.

For all of the six guns tested, and in every cut-off condition, the precision obtained on the whole curve is better than $5 \%$ which is far better than the results obtained by the previous studies, even based on numerical codes.

\section{Complementary observations}

First, for all of our guns, the influence of the third grid (G3) on the electric field (and, as a result, on the intensity) is negligible. However, we can easily imagine that for guns with large-hole-grids, and small gaps between the grids, this influence would be a bit more significant. 
Then, for $\mathrm{V}_{\mathrm{K}}=0 \mathrm{~V}$, the ratio between the intensity, or the current density, or $\mathrm{V}_{\mathrm{G} 2}$ (at cut-off), or the electric field, in two different cut-off conditions, is independent of the gun. For example, for every gun, between $6 \mathrm{MIK}$ and $7 \mathrm{MIK}$ conditions, we have:

$$
\begin{gathered}
\text { For any }(\mathrm{x}, \mathrm{y}), \frac{I_{O V}^{7 M I K}}{I_{O V}^{6 M I K}}=\frac{j_{0 V}^{7 M I K}(x, y)}{j_{O V}^{6 M I K}(x, y)}=5.2, \\
\text { and: } \frac{V_{G 2 c o}^{7 M I K}}{V_{G 2 c o}^{6 M I K}}=\frac{E_{0 V}^{7 M I K}(x, y)}{E_{O V}^{6 M I K}(x, y)}=3 .
\end{gathered}
$$

Indeed, in cut-off conditions (i.e. at the limit of emission), the cathode electric field is zero, and as $\mathrm{V}_{\mathrm{Gl}}=0$ and $\mathrm{V}_{\mathrm{G} 3}$ has a week influence, from formula (1) we have:

$$
\begin{aligned}
& 0=V_{K c o} \cdot E_{K}\left(V_{K}=1 V, V_{G 1}=V_{G 2}=V_{G 3}=0\right) \\
& +V_{G 2 c o} \cdot E_{K}\left(V_{G 2}=1 V, V_{K}=V_{G 1}=V_{G 3}=0\right)
\end{aligned} .
$$

Therefore, for two cut-off configurations (1) and (2), we get for every gun:

$$
\frac{V_{G 2 c o}^{(1)}}{V_{G 2 c o}^{(2)}}=\frac{V_{K c o}^{(1)}}{V_{K c o}^{(2)}}=\text { constant. }
$$

Moreover, at $\mathrm{V}_{\mathrm{K}}=0 \mathrm{~V}$ :

$E_{K}\left(V_{K}, V_{G 1}, V_{G 2}, V_{G 3}\right)=V_{G 2} \cdot E_{K}\left(V_{G 2}=1 V, V_{K}=V_{G 1}=V_{G 3}=0\right)$

Then, for two cut-off configurations (1) and (2):

$$
\begin{aligned}
& \frac{I_{0 V}^{(1)}}{I_{0 V}^{(2)}}=\frac{j_{0 V}^{(1)}(x, y)}{j_{0 V}^{(2)}(x, y)}=\left(\frac{V_{G 2 c o}^{(1)}}{V_{G 2 c o}^{(2)}}\right)^{3 / 2} \\
& =\left(\frac{V_{K c o}^{(1)}}{V_{K c o}^{(2)}}\right)^{3 / 2}=\left(\frac{E_{0 V}^{(1)}(x, y)}{E_{0 V}^{(2)}(x, y)}\right)^{3 / 2}=\text { constant. }
\end{aligned}
$$

\section{PERSPECTIVES: ADAPTATION OF THE MODEL FOR NEW GUNS}

For studying new guns, when no experimental reference at $\mathrm{V}_{\mathrm{K}}=0 \mathrm{~V}$ is available, we can use Ploke's equivalent diode distance concept to determine parameter $\mathrm{D},{ }^{10}$ and obtain drive curves with a precision quite satisfying, but of course lower than the one in the last paragraph. As recalled by H. Suzuki, ${ }^{21}$ in a rotationally symmetrical electron gun, the equivalent diode distance can be written:

$$
D_{e}=\frac{V_{K c o}}{E_{\text {max }}\left(V_{K}=0 V\right)}
$$

When using this definition, and here, without any ChildLangmuir's law correction, for gun al (the best case), we already have an acceptable coherence with the experiment (see figure 12).

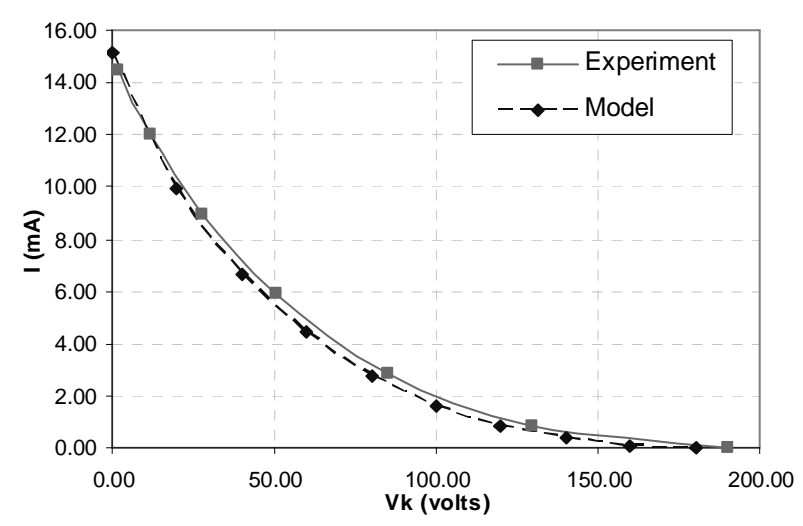

FIG. 12. Drive curve for gun $\mathrm{a} 1, \mathrm{~V}_{\mathrm{K} \text { co }}-190 \mathrm{~V}$, and $\mathrm{D}-0.29 \mathrm{~mm}$.

We notice that for high intensities, the model describes very well the experiment, unlike for small intensities, where we have a factor 2 between the two curves.

As a result, we have to modify a little the definition of the correction so as not to correct the drive curve for high intensities. The simplest solution appeared to be the conservation of the previous correction divided by its value at maximum intensity. For instance, in the case of gun a1, we have to divide the correction calculated in the previous chapter by 1.3. Finally, we obtain for gun a1 the curves displayed in figure 13.

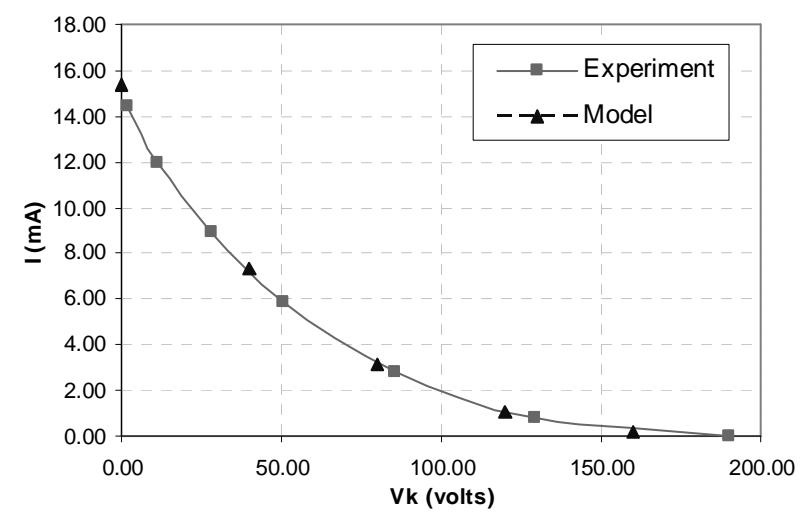

FIG. 13. Drive curve for gun $\mathrm{a} 1, \mathrm{~V}_{\mathrm{K}}$ co $-190 \mathrm{~V}$, and $\mathrm{D}-0.29 \mathrm{~mm}$.

Without any experimental reference, the model precision is therefore very high.

Moreover, amoung our six tested guns, four guns present the same coherence. For the other two, the precision is $9 \%$ for high currents $(>4 \mathrm{~mA})$ and $20 \%$ for low currents $(<1 \mathrm{~mA})$.

\section{CONCLUSION}

We have first proposed a new and precise analytical model of current generation for rotationally symmetrical electron guns that provides a better accuracy on the whole intensity-voltage characteristic curve than the simulation codes. This model uses only the Laplace field on the cathode, which can be obtained easily with an analytical procedure. Moreover, the finite radius of the beam is taken into account by correcting the classical Child-Langmuir's law, and in addition, an equivalent diode gap parameter is defined. 
Then, we have generalized this study to a fully three dimensional model, valid for all kinds of electron gun geometries. The only numerical input needed is again the Laplace electric field at the cathode in elementary conditions. The precision obtained is far better than the one of the simulation codes encountered (accuracy better than $5 \%$ on the whole drive curve domain).

Even for new guns in design process, by using Ploke's equivalent diode distance concept, we can get a quite satisfying precision on the whole intensity-voltage characteristic curve.

Finally, we have managed to understand the main mechanisms at stake in the electron gun current generation. And, coupled with investigations on the electron beam dynamics, such as source emittance modelling, this study provides a good reproduction of the electron gun physics.

\section{ACKNOWLEDGEMENT}

This work was performed under the scope of a $\mathrm{PhD}$ thesis, sponsorized and supported by Thomson.

\section{APPENDIX}

We consider a system made of several coaxial cylindrical electrodes whose radius is denoted R. We suppose the gap between electrodes to be small. The purpose of this section is to give a simple analytical model for the calculation of the potential. It is an alternative solution to numerical methods (like finite elements), and is general, accurate, and avoids any modelling of the electrodes.

The radial position is denoted " $\mathrm{r}$ " and the longitudinal one is denoted " $z$ ".

We look for a fundamental solution $\mathrm{F}$ of the cylindrical Laplace problem such as:

$$
F(r, z)=f(\omega r) \exp (j \omega z)
$$

This function verifies the Laplace equation $\Delta \mathrm{F}=0$ if

$$
f=\frac{1}{r} \frac{\partial}{\partial r}\left(r \frac{\partial f}{\partial r}\right)
$$

A solution is the Bessel function $\mathrm{I}_{0}$.

Consequently, we look for a general potential $\mathrm{V}$ such as:

$$
V(r, z)=\int_{-\infty}^{+\infty} K(\omega) I_{0}(\omega r) \exp (j \omega z) d \omega
$$

We suppose the potential known on the boundaries ( $\mathrm{r}=\mathrm{R})$. It is constant on the electrodes and we can suppose with a good approximation that the potential varies linearly between 2 successive electrodes.

We have:

$$
V(R, z)=\int_{-\infty}^{+\infty} K(\omega) I_{0}(\omega R) \exp (j \omega z) d \omega
$$

The goal is to get the kernel $\mathrm{K}$ from the known function $\mathrm{V}(\mathrm{R}, \mathrm{z})$. The previous integral is a Fourier transform. A simpler form of the problem is to consider the derivative of $\mathrm{V}$ versus $\mathrm{Z}$ on the cylindrical boundary. This function is constant in the gaps (equal to the gap voltage divided by the gap length) and zero on the electrodes. This function is hence a combination of Heaviside distributions (see figure 14).
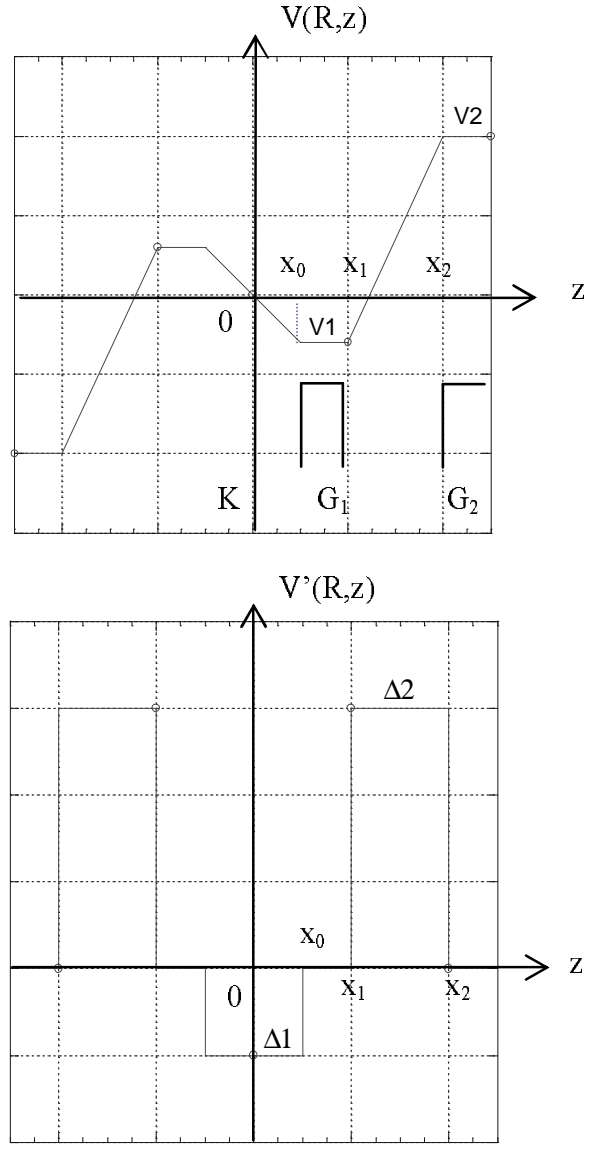

FIG. 14. Potential on boundary (top) and its derivative (bottom), combination of Heaviside distributions. We solve the bottom problem and we get the top one by integration.

The fundamental problem to be solved is the normalized $(\mathrm{R}=1)$ Heaviside problem:

$$
H(z)=\int_{-\infty}^{+\infty} K_{h}(\omega) I_{0}(\omega) \exp (j \omega z) d \omega
$$

By taking the inverse Fourier transform of $\mathrm{H}$, one gets:

$$
K_{h}(\omega) I_{0}(\omega)=\frac{\delta}{2}+\frac{j}{2 \pi \omega}
$$

We have the following approximation:

Hence:

$$
I_{0}(\omega) \approx 1+\frac{\omega^{2}}{4}+\frac{\omega^{4}}{64}
$$

Finally, we get:

$$
K_{H}(\omega) \approx \frac{\frac{\delta}{2}+\frac{j}{2 \pi \omega}}{1+\frac{\omega^{2}}{4}+\frac{\omega^{4}}{64}}
$$

$K_{H}(\omega) I_{0}(\omega \rho) \approx \frac{1+\frac{(\omega \rho)^{2}}{4}+\frac{(\omega \rho)^{4}}{64}}{1+\frac{\omega^{2}}{4}+\frac{\omega^{4}}{64}}\left(\frac{\delta}{2}+\frac{j}{2 \pi \omega}\right)=N(\omega, \rho)$ 
The potential is obtained as follows:

- A Fourier transform of $\mathrm{N}$ is calculated, which is easily carried out by tools like Maple or Mathematica, leading to a reference function $M_{0}(\rho)=\hat{N}(\omega, \rho)$ (where the symbol ${ }^{\wedge}$ denotes the Fourier transform). The "zero" index indicates the Heaviside problem at origin.

- The Heaviside problem at any location $\mathrm{z}_{\mathrm{i}}$ is the translation of the previous function and is written $M_{i}(\rho)$.

- The weight of each Heaviside distribution is the ratio $\Delta \mathrm{V} / \mathrm{g}$ where $\Delta \mathrm{V}$ is the voltage gap and $\mathrm{g}$ the electrode gap.

- As the integral of $\mathrm{M}_{0}$ is easily obtained analytically, the general solution is obtained from the previous steps.

The general procedure given here is implemented in a few lines of Maple language. We check that the approximation made on the $\mathrm{I}_{0}$ function gives a good enough precision.

${ }^{1}$ M. H. L. M. van den Broek, J. Appl. Phys. 60 (11), 3825-3835 (1986a)

${ }^{2}$ D. M. MacGregor, IEEE Trans. Consum. Electron. CE-33 (8), 10981106 (1986).

${ }^{3}$ D. J. Bechis, J. R. Fields, D. A. New, W.B. Paul, E. R. Sverdlov, N. D. Winarsky, T. C. Lausman, Proc. SID 30 (3), 229-239 (1989).

${ }^{4}$ A. F. Aalders, J. Van Engelshoven, J. Gerritsen, SID Intern. Symp. Digest, XX, 38-41 (1989).
${ }^{5}$ R. C. Alig, J. R. Fields, Journal of the Society for Information Display 8, Issue 2, 99-113 (2000).

${ }^{6}$ J. Petillo et al., IEEE Transactions on Plasma Science 30, no. 3, 12381264 (2002).

${ }^{7}$ H. Moss, Pt. II, J. Brit. iRE 6, 99-129 (1946).

${ }^{8}$ H. Moss, Academic Press, News York, 15-26 (1968b).

${ }^{9}$ M. Ploke, Z. Angew. Phys 3, 441-449 (1951).

${ }^{10}$ M. Ploke, Z. Angew. Phys. 4, 1-12 (1952).

${ }^{11}$ R. D. Gold, J. W. Schwartz, RCA Rev. 24, 564-583 (1958).

${ }^{12}$ J. C. Francken, Philips Tech. Rev. 21, 10-23 (1959/60).

${ }^{13}$ J. Bessho, NHK (Jpn. Broadcast. Corp.) Tech. Rep. 15, 233-254 (1963).

${ }^{14}$ J. Hasker, Philips Res. Rep. 21, 122-150 (1966).

${ }^{15}$ J. Hasker, Philips Res. Rep. 27, 513-538 (1972).

${ }^{16}$ A. A. van Gorkum, Optik 71 (3), 93-104 (1985).

${ }^{17}$ M. H. L. M. van den Broek, J. Phys. D: Appl. Phys. 19, 1389-1399 (1986b).

${ }^{18}$ C. D. Child, Phys. Rev. 32, 492 (1911); I. Langmuir, ibid. 2, 450 (1913).

${ }^{19}$ J.M. De Conto, R. Poux, «Modélisation d'un canon à électrons de tube cathodique », LPSC internal report, 3-21, 2003 (unpublished).

${ }^{20}$ W.S. Koh, L.K. Ang and T.J.T. Physics of Plasmas 12, 053107, 1-6 (2005).

${ }^{21}$ H. Suzuki, Imaging and Electron Phys. 105, 283-284 (1999). 